\title{
PARO JUVENIL EN LA REPUBLICA FEDERAL DE ALEMANIA Y ESPAÑA
}

\section{Manfred Wallenborn}

RESUMEN. Se ofrece una comparación entre la República Federal de Alemania y España respecto de la cuantía y distribución del paro juvenil, así como de las políticas de formación y empleo. En la comparación resulta más angustiosa y preocupante la situación española. El detallado análisis de diferencias y puntos comunes arroja automáticamente importantes sugerencias para una mejora de la política de empleo juvenil. Especialmente preocupante es el cuadro de la mujer española, tanto en relación con su evolución en las estadísticas del desempleo como en la discriminación que sufre en el acceso a las medidas de reciclaje y formación.

Este artículo quière ser un resumen actualizado de mi libro Paro Juvenil en la República Federal de Alemania y España. Estudio Comparado a partir de las respectivas soluciones estatales ${ }^{\mathrm{I}}$. Este trabajo de más de 300 páginas se terminó en la primavera de 1986. En consecuencia, sólo ha podido basarse en datos y estadísticas disponibles hasta finales de 1985 .

Quiero ofrecer aquí las principales ideas y conclusiones del mencionado estudio. En la medida de lo posible, me ocuparé también de la evolución actual y señalaré, además, algunas lagunas existentes en el campo de la investigación.

En el trabajo aludido se dedica la misma extensión a las problemáticas de ambos países. En este artículo me ocuparé, sin embargo, con mayor detalle de

${ }^{1} \mathrm{M}$. WALLENBORN, Jugendarbeitslosigkeit BRD/Spanien. Ein Vergleich unter Beruecksichtigung staatlicher Loesungsansaetze, Francfort, 1987. 
Alemania y de los intentos de solución a su problema del paro juvenil. Ello es debido al hecho de que el lector conoce seguramente mejor la situación española y que, además, prefiera echar una ojeada a lo que pasa allende sus fronteras. En aras de una visión lo más sinóptica y completa posible, se ha prescindido en esta ocasión de un gran cúmulo de datos, estadísticas y tablas que aparecen en el libro.

\section{SOBRE EL VERDADERO PORQUE DE UNA COMPARACION ENTRE LOS DOS PAISES}

La razón estriba no sólo en el hecho de que en ambos países la magnitud, características y consecuencias del paro juvenil se hayan convertido en un tremendo problema social. Se debe, sobre todo, a la circunstancia de que en ambos países son bastante diferentes las diversas medidas aplicadas a la solución del problema. En efecto, mientras que en la República Federal de Alemania aquellas medidas se centran casi exclusivamente en el capítulo de la formación, predominan en España de modo sobresaliente las medidas encaminadas al fomento directo del empleo. Aquí radica, ciertamente, una diferencia básica entre ambos países. Por ello, la comparación debería aclarar las siguientes consideraciones:

- Dentro de cada país, qué medidas están contribuyendo más eficazmente al alivio del paro juvenil.

- Si los correspondientes programas son, en realidad, suficientes para combatir el paro juvenil.

- Si aquellas medidas garantizan a los implicados una fundada perspectiva de colocación, es decir, si proporcionan una cualificación suficiente para asegurar a la larga un puesto de trabajo.

- Si los distintos grupos de jóvenes en situación precaria se encuentran suficientemente representados en el paquete de medidas.

Para responder a estas preguntas fueron precisos algunos trabajos preliminares. Se necesitó determinar más en detalle la magnitud, la estructura y las causas del paro juvenil en ambos países. El paso siguiente consistió en presentar las medidas más importantes aplicadas en cada país y valorarlas en base a la literatura disponible y diversos datos secundarios. Con el fin de esclarecer los resultados de la parte teórica y, sobre todo, valorar la efectividad de las políticas en un espacio social limitado, se realizó en cada país una investigación empírica, que indagaba el paradero y las perspectivas de colocación de los afectados.

Pese a que, desde un principio, cada país proyectaba semejante investigación, fue necesario acometerla por nuestra parte. Sobre todo, en relación con 
España disponía de muy pocas publicaciones acerca del ulterior paradero de los participantes en los mencionados programas. Hasta donde pude averiguar, se contaba hasta entonces con un trabajo de L. Cachón Rodríguez sobre dicha temática ${ }^{2}$.

\section{PUNTOS COMUNES, PARECIDOS Y DIFERENCIAS \\ EN LA PROBLEMATICA DEL PARO JUVENIL EN AMBOS PAISES}

E1 paro juvenil se diferencia fundamentalmente en cuanto a su cuantía. En España, aproximadamente, uno de cada dos parados es o era joven (aproximadamente, 1.400 .000 en el tercer trimestre de 1986). En la República Federal de Alemania, uno de cada cuatro parados tiene menos de 25 años (503.000 personas, es decir, 24,6 por 100 de los parados en septiembre de 1986). Son datos oficiales de ambos países ${ }^{3}$.

No pueden hacerse aquí consideraciones más amplias sobre la validez de estos datos oficiales o sobre las cifras ocultas y la reserva potencial. El estudio antes mencionado puede avalar, sin embargo, que, en 1982, la cifra oficial de los jóvenes alemanes parados o en busca de formación sólo constituía un 51,4 por 100 de la juventud realmente desempleada ${ }^{4}$. Meramente el hecho de que en ambos países haya aumentado la cifra total de ocupados, mientras que el paro se haya modificado casi inapreciablemente, apunta a la existencia de una importante reserva potencial en el mercado de trabajo. Ilustra también la circunstancia de que los grupos de ese segmento demandan de nuevo una colocación ${ }^{5}$.

El paro juvenil sigue en ambos países las peripecias del paro global. $\mathrm{Si}$ éste - como ocurre en la República Federal de Alemania- desciende ligeramente, también descenderá el paro juvenil en parecida proporción. Así, aún hoy (cifras de septiembre de 1986), de cada cuatro parados uno es joven.

En la República Federal de Alemania deben contemplarse junto con los jóvenes parados aquellos otros en busca de formación. Las especiales características de la formación (sistema dual) afecta en Alemania a un 60 por 100

${ }^{2} \mathrm{~L}$. CACHón Rodríguez, L'insertion professionelle des jeunes en Espagne pendant la crise, Vaucresson, 1985.

${ }^{3}$ Bundesanstalt fuer Arbeit, Amtliche Nacbrichten der Bundesanstalt tuer Arbeit, Bestand an Arbeitslosen und offenen Stellen nacb ausgewaeblten Strukturmerkmalen, núm. 3, de 31-3-1987, Nuremberg, 1987, pp. 174 y ss., y Ministerio de Trabajo y Seguridad Social, Avance, núm. 16, enero 1987, Madrid, p. 7.

- Cfr. M. Wallenborn, op. cit., p. 24. También, el libro de H. J. Petzold/W. Sc h leGEL, Qual obne Wabl, Jugendliche zwischen Scbule und Beruf, Francfort, 1983.

${ }_{5}$ Para una ilustración de los diferentes segmentos del mercado de trabajo, cfr. H. Bie HLER y otros, Arbeitsmarktstrukturen und -prozesse. Zur Funktionsweise ausgewaeblter Arbeitsmaerkte, Tubinga, 1981. 
de cada cohorte ${ }^{6}$. El organismo Bundesanstalt fuer Arbeit (homólogo del INEM español) no considera parado a quien procura una plaza de formación en los sectores privado o público porque stricto sensu no buscan directamente empleo, sino simplemente una plaza de formación.

Muchos jóvenes alemanes tienen importantes problemas en el llamado «primer umbral» (paso de la escuela a un período de aprendizaje) porque no encuentran una oportunidad de formación en la profesión deseada o incluso en ninguna parte. Sin embargo, es de esperar cierto alivio en este particular gracias a la modificación de la estructura demográfica, la extensión de las medidas del gobierno y a la mayor disponibilidad de oportunidades (a menudo de dudosa categorización) en el sistema dual. Al mismo tiempo se han agudizado, sin embargo, los problemas propios del «segundo umbral» (paso de la formación a la colocación). Los mismos, tanto de orden cuantitativo como cualitativo, se han trasladado a otro campo, el de la reducción del períado de formación.

En base a una política casi exclusivamente orientada desde los inicios de los ochenta a ampliar el número de plazas de formación, muchos jóvenes han sido entrenados en oficios relativamente poco cualificados (duración de dos años en lugar de tres) o de escasas perspectivas de colocación. La consecuencia ha sido el paro tras la aplicación de tales medidas de formación y reciclaje. De acuerdo con los resultados de un sondeo representativo del Instituto Federal de Formación Profesional (BIBB), la situación era la siguiente seis meses después de finalizar el período de formación:

- 51 por 100 de los encuestados se encontraban colocados en consonancia con la formación seguida;

- 28 por 100 se encontraban colocados en un sector distinto al que su formación les destinaba, o bien tenían que ampliar su formación;

- 10 por 100 se encontraban en paro ${ }^{7}$.

En la República Federal de Alemania es, sobre todo, la artesanía, o sea, pequeñas empresas más interesadas en una mano de obra barata que en una adecuada formación del personal, las que contribuyen al mencionado desarrollo. Hoy en día se movilizan considerables recursos para ofrecer a los jóvenes una aceptable perspectiva de empleo después de haber seguido una formación con muy dudosas posibilidades de colocación. Me refiero a la formación para oficios tales como panadería, mecánica de vehículos, jardinería, peluquería, ventas, etc. ${ }^{8}$. La Oficina Federal del Trabajo aporta estos fondos.

- Un buen informe sobre la evolución del sistema dual lo ofrecen las reseñas anuales del Ministerio de Educación y Ciencia (BMBW), en Bonn.

${ }^{7}$ Bundesinstitut fuer Berufsbildung, Berufsausbildung abgeschlossen, was dann? Ergebnisse einer Laengsschnittuntersuchung, Berlín, 1987, p. 20.

${ }^{8}$ H. Stegmann, "Wird die "zweite Schwelle" immer hoeher?", en M. KaISER y otros, Berufliche Verbleibforschung in der Diskussion Materialienband, 1, Nuremberg, 1985. 
En España se da, en parte, una composición diferente del agregado de los parados juveniles, encontrándose especialmente afectados los menores de 20 años.

Jóvenes parados (en miles)

\begin{tabular}{|c|c|c|}
\hline \multirow{3}{*}{$\begin{array}{lllllll}16-19 & \text { años } & \ldots & \ldots & \ldots & \ldots & \ldots \\
20-24 \text { años } & \ldots & \ldots & \ldots & \ldots & \ldots & \ldots\end{array}$} & España ${ }^{9}$ & $R F A^{10}$ \\
\hline & $\begin{array}{l}498,2 \\
882,6\end{array}$ & $\begin{array}{l}149,0 \\
354,2\end{array}$ \\
\hline & $\boldsymbol{\Sigma}=1.380,8$ & $\boldsymbol{\Sigma}=503,2$ \\
\hline
\end{tabular}

La diferencia de distribuciones de ambos países tiene que ver con las medidas aplicadas. En la República Federal de Alemania, la política de extensión del período de formación en el llamado primer umbral ha conseguido «aparcar» un considerable número de jóvenes; es decir, éstos no figuran en las estadísticas de jóvenes que buscan empleo o una plaza de formación. Esta misma tendencia se ve reforzada en la República Federal de Alemania por una más prolongada y frecuente permanencia de jóvenes menores de 20 años en las escuelas de formación. Por el contrario, España registra, entre los países de la OCDE, la tasa más baja de este tipo de formación referida a jóvenes menores de 20 años ${ }^{11}$.

En el mercado de trabajo de ambos países se dan varios grupos nítidamente diferenciados. Cabe aquí hacer especial mención de la situación de la mujer, tanto en Alemania como en España. En el primer país hay que añadir el grupo de los jóvenes extranjeros. Pese a que en ambas naciones ha aumentado precisamente el asentamiento de mujeres jóvenes en el sistema de formación, aliviando así un tanto la situación laboral, es preciso apuntar la existencia de altas tasas de paro específicas por sexo. Esta circunstancia nada tiene que ver con la cualificación de las mujeres paradas. Estas están tanto o más cualificadas que los chicos. Simplemente se trata de una clara discriminación ante la mujer en ambos países. En Alemania se da, además, el hecho de que los chicos son preferidos en la contratación para puestos de formación mejor cualificados. Igualmente, tienen mayores probabilidades de encontrar empleo después de terminado el período de formación. He aquí algunos datos:

9 Ministerio de Trabajo..., Bundesanstalt fuer Arbeit, op. cit.

${ }^{10}$ Idem.

$" \mathrm{M}$. Beltrán Villalba y otros, Informe Sociológico sobre la Juventud Española 1960/82, Madrid, 1984, p. 55. 
Parados jóvenes (en miles) por edad y sexo

\begin{tabular}{|c|c|c|c|c|}
\hline & \multicolumn{2}{|c|}{ España ${ }^{12}$} & \multicolumn{2}{|c|}{$R F A^{13}$} \\
\hline & Hombres & Mujeres & Hombres & Mujeres \\
\hline \multirow[t]{2}{*}{ 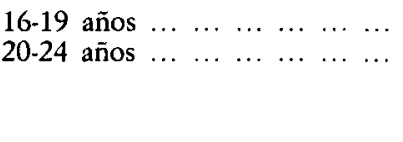 } & $\begin{array}{l}265,2 \\
482,2\end{array}$ & $\begin{array}{l}233,0 \\
400,4\end{array}$ & $\begin{array}{r}66,0 \\
166,1\end{array}$ & $\begin{array}{r}83,0 \\
188,2\end{array}$ \\
\hline & $\boldsymbol{\Sigma}=747,4$ & $\Sigma=633,4$ & $\Sigma=232,1$ & $\boldsymbol{\Sigma}=271,2$ \\
\hline
\end{tabular}

En cuanto al aspecto meramente formal de la formación obtenida - con ello no se prejuzga, sin embargo, nada sobre la utilidad práctica de las cualificaciones obtenidas en el período de formación-, la mayoría de los jóvenes españoles acaban mejor cualificados. Esto puede ilustrarse teniendo en cuenta la proporción relativamente alta de jóvenes parados que adquieren un título medio o medio alto. Por el contrario, el paro de titulados superiores permanece muy por debajo de la media general. Al mismo tiempo, más de la mitad de todos los jóvenes parados alemanes no poseen una formación completamente terminada, lo cual se repite en la estructura de cualificaciones del paro global.

La delicada situación de los jóvenes españoles frente al sistema de empleo se refleja no sólo en el indicador antes comentado. Se repite teniendo en cuenta la duración del paro; 34 por 100 de los jóvenes parados lo sufren ya al menos dos años ${ }^{14}$. Ello hace presumir un endurecimiento estructural del mercado de trabajo. En Alemania, en cambio, sólo un 5 por 100 de los jóvenes ha permanecido tanto tiempo en esa situación de desempleo ${ }^{15}$. Lo efímero del paro es precisamente una de las características de los jóvenes alemanes, que es también propia del sistema dual: concluido el período de formación, las empresas no están obligadas a contratar a esos jóvenes. Sin embargo, el personal bien cualificado acaba por encontrar trabajo después de un breve período de desempleo. Puede que hayan de contentarse con una colocación por debajo de su preparación. Los que, una vez concluida su formación, no encuentran trabajo en su sector profesional acostumbran a acogerse a otras medidas de extensión de la formación o reciclaje en otro campo, eludiendo así el registro en la lista de parados. Se comprende así que, a tenor de un amplio sondeo en septiembre de 1986, un 53,4 por 100 de los parados alemanes de menos

\footnotetext{
${ }_{12}$ Ministerio de Trabajo... y Bundesanstalt..., op. cit.

${ }^{13}$ Idem.

${ }^{14}$ El País del 1 de junio de 1987.

15 Bundesanstalt fuer Arbeit, Amtlicbe Nacbricbten..., op. cit., p. 265.
} 
de 25 años sólo permanecían parados un máximo de tres meses. Sólo un 12,9 por 100 se encontraba en tal situación más de un año ${ }^{16}$.

Cabe mencionar aquí algunas razones que explican estas diferencias en la segmentación del mercado de trabajo de los jóvenes en ambos países. De cara a las escasas nuevas colocaciones que se registran en España, son preferidos, ante todo, aquellos candidatos con cargos familiares o con experiencia profesional. De ello se deducen limitaciones de acceso para aquellos jóvenes que buscan el primer empleo. Por el contrario $-\mathrm{y}$, en parte, por parecidas razones-, se recurre con menos frecuencia al despido de empleados más adultos, habida cuenta que los costes de su despido aumentan con la acumulación de años de pertenencia a la empresa.

En la República Federal de Alemania se ha seguido una política de licenciamiento de personal mediante jubilaciones anticipadas en varios años. Todo ello, y el hecho de que sean los más jóvenes los mejor cualificados en el manejo de nuevas tecnologías, abren a éstos unas perspectivas de empleo relativamente más halagüeñas. Mediante una aplicación flexible de la legislación laboral se da la posibilidad de un empleo a tiempo limitado, como también ocurre en España. Las empresas recurren a menudo a esta contratación temporal. Dado que esta regulación es en Alemania relativamente reciente, apenas se dispone de experiencias sobre la efectividad de esta forma de empleo temporal. En esta línea deberían organizarse estudios ulteriores.

Cabe aducir para ambos países una serie de causas adicionales que explican la magnitud y naturaleza del paro juvenil en ambos países. Aquéllas son de tipo estructural, aunque también coyuntural, demográfico y regional. Todo ello sustenta la precaria situación de los jóvenes. Toda esta temática se sale del marco de este trabajo, que debe centrarse, en cambio, en la valoración de las medidas diseñadas para combatir el paro juvenil. No se investigarán ni valorarán aquí aquellas medidas específicamente regionales o propias de las Comunidades Autónomas o de los Estados regionales de Alemania. Se ha estudiado tan sólo los programas más característicos e importantes a nivel nacional.

Para el caso de España se trataría, pues, de seleccionar y evaluar las siguientes medidas:

- contratación temporal,

- contratación a tiempo parcial,

- contratos en prácticas,

- contratos para la formación.

Sobre este particular se disponía en la literatura española de datos hasta finales del año $1984^{17}$.

${ }^{16}$ Bundesanstalt fuer Arbeit, op. cit., p. 265.

${ }_{17}$ Cfr., por ejemplo, Ministerio de Trabajo y Seguridad Social, Empleo y paro en Es- 
En cuanto a la República Federal de Alemania se han presentado y valorado aquellas ofertas destinadas a una formación cualificada en el sistema dual, así como a las que, sin contener un programa de cualificación, preparan a los jóvenes a asumir una relación de empleo ${ }^{18}$. Unas medidas se asientan, pues, en el sistema de empleo; otras, en el sistema de formación. Todo ello está en correspondencia con la lógica de la política oficial, que pretende superar el problema del paro (juvenil) con una ofensiva en el campo de la formación o, mejor dicho, de la cualificación. Cabe trazar una serie de diferencias, puntos comunes y parecidos entre ambos países, según se expondrá de modo global a continuación.

\section{Diferencias}

En primer lugar, ambos países difieren entre sí respecto de la ubicación de las medidas. En España no se dan, como en Alemania, programas de carácter casi exclusivamente de formación. En este último país se afirma reiteradamente la prioridad de una adecuada formación para el sistema de empleo, mientras que una buena parte de las medidas españolas están destinadas a colocar directamente a los jóvenes. Incluso los esfuerzos de cualificación del personal se instalan en España preferentemente en el mismo sistema de empleo, mientras que en la República Federal de Alemania se recurre fundamentalmente a las escuelas y otras instituciones de extensión de la formación.

Las ofertas de cualificación están más diferenciadas en Alemania en función de los distintos grupos específicos sometidos a tratamiento. La consigna es aplicar a cada grupo sus propias medidas. En consecuencia, a algunos jóvenes se les aplican medidas más bien cuestionables y que tienen influencia decisiva en el ulterior desarrollo profesional. Esta mayor diferenciación se explica por la historia y la tradición. En parte, estas medidas existen hace quince años y prosiguieron a raíz de la crisis en el mercado de trabajo.

España reaccionó —en comparación con la República Federal de Alemania- relativamente tarde a los problemas del paro juvenil. Esto puede ilustrarse teniendo en cuenta la naturaleza y amplitud de la oferta ${ }^{19}$. Sólo a partir de 1982 se registra una rápida ampliación del paquete de medidas; desde entonces se asiste a la promulgación de diversas disposiciones legales de aplicación práctica con ayuda del INEM. Nuestro trabajo se resiente, por consiguiente, del hecho de que había que disponer de un time-lag de dos o más años para proceder a la evaluación de las correspondientes acciones y cifras.

paña durante 1984, Madrid, 1985. También, Ministerio de Trabajo y Seguridad Social, Mercado de Trabajo en España durante 1985, Madrid, 1986.

${ }_{18}$ Para más detalles, cfr. M. Wallenborn, Jugendarbeitslosigkeit..., op. cit.

${ }^{19}$ Cfr. al respecto, también, Ministerio de Cultura, Revista de la Juventud, núm. 15 , Madrid, 1984. 
Otras diferencias se refieren a los organismos competentes en la materia. Mientras que en España es la Administración del Trabajo el órgano competente en las cuatro ofertas señaladas, en la República Federal de Alemania esta circunstancia se da sólo parcialmente. En este país una buena parte de las medidas se llevan a cabo en las escuelas de formación (el organismo competente es aquí el gobierno o ministerio de cultura de los Estados regionales) porque así cabe diseñar formas de cualificación más baratas, aunque también más cuestionables. Además, y merced a la introducción de un año escolar obligatorio adicional y de clases encaminadas a la preparación profesional del joven, el sistema de educación pudo reaccionar en la forma más rápida y económica ante el problema de la escasez de puestos, tanto en el mercado de trabajo como en el de la formación. Con ello se vieron aliviadas considerablemente las estadísticas de la administración laboral; en efecto, los jóvenes que seguían en las escuelas no contaban como parados o carentes de una plaza de formación.

También respecto de la duración se diferencian considerablemente las medidas aplicadas en ambos países. Mientras que en España algunas medidas pueden durar sólo tres o seis meses, haciéndose fuertes concesiones a los empresarios en el capítulo de la flexibilidad, en Alemania aquellos programas duran casi siempre un mínimo de un año. Pueden llegar hasta los tres años cuando se trata de una formación profesional cualificada.

El hecho de que precisamente en España muchas pequeñas empresas recurren a medidas tales como la de contratación para la formación, disponiendo, además, de un gran margen de flexibilidad, despierta la sospecha de que se interesan más bien por una mano de obra joven subvencionada. Por lo mismo, apenas desempeñan un papel los componentes de cualificación o una prolongada integración en el sistema de empleo. Algunos estudios empíricos han podido ratificar parcialmente esta sospecha, según se expondrá luego. En la República Federal de Alemania, los jóvenes que con el apoyo del Estado han terminado el período de formación (el llamado programa de atención a los desfavorecidos) tienen peor suerte en el mercado de trabajo que quienes han cumplido un tiempo de formación dentro de la empresa. Como contrapunto a la investigación del Instituto Federal de Formación Profesional (BIBB), basado en un estudio transversal representativo de todos los jóvenes y destinado a averiguar su paradero después de la formación, Petzold y Schlegel ${ }^{20}$ investigaron únicamente la suerte sufrida por los egresados del programa de atención a los desfavorecidos. He aquí los resultados:

${ }^{20}$ H. J. Petzold /W. Schlegel, «Die Zweite Schwelle als Stolperstein?», en Materialien und Praxisbericbte zur Ausbildung und Arbeit von Jugendlicben, tomo 5, Heidelberg, 1986. 
Destino profesional de los egresados del programa de atención a los desfavorecidos

\begin{tabular}{|c|c|c|c|c|c|}
\hline & $\begin{array}{l}\text { Protesión } \\
\text { aprendida }\end{array}$ & $\begin{array}{c}\text { Profesión } \\
\text { afin }\end{array}$ & $\begin{array}{l}\text { Ocupación } \\
\text { sin } \\
\text { cualificar }\end{array}$ & Paro & $\begin{array}{c}\text { Otro } \\
\text { paradero }\end{array}$ \\
\hline Tatal de egresados ... & 40 & 16 & 8 & 27 & 9 \\
\hline $\begin{array}{llll}\text { Hombres } & \ldots & \ldots & \ldots\end{array}$ & 45 & 18 & 8 & 17 & 12 \\
\hline Hombres extranjeros. & 56 & 23 & 5 & 8 & 7 \\
\hline Mujeres $\ldots \ldots \ldots \ldots$ & 31 & 11 & 9 & 43 & 5 \\
\hline
\end{tabular}

La crítica lanzada a las medidas alemanas se refieren fundamentalmente al hecho de que su misma diferenciación redobla las deficiencias de formación y la discriminación social anteriores, es decir, a los menos formados se les aplica medidas de menor cualificación. Ciertamente, es posible el paso de un nivel a otro superior. Sin embargo, se registran muchos abandonos prematuros. Estos jóvenes suelen optar, antes del término de aplicación de las medidas, por una ocupación no cualificada, estando expuestos más tarde a nuevas y más graves crisis del mercado de trabajo. En efecto, varias previsiones coinciden en afirmar que el porcentaje del personal no cualificado en el total pasará de 30 a 20, entre hoy y el año 2000, en la República Federal de Alemania.

Por otra parte, no se comprende que haya medidas encaminadas tan sólo a conseguir un trabajo y no a preparar a la gente en el sentido profesional. La política de las Oficinas de Empleo de ubicar a los jóvenes en ofertas que no proporcionan entrenamiento profesional alguno, o no los preparan en tal sentido, merma las posibilidades de iniciar una actividad con cierta cualificación. Los expertos están denunciando desde hace una década la problemática aludida ${ }^{21}$.

Finalmente, se dan diferencias respecto de la representación de las chicas. En España dominan —excepción hecha de la contratación a tiempo parciallos chicos de forma abrumadora, en tanto que en la República Federal de Alemania las mujeres están bien representadas, e incluso a partes iguales con el varón. Sin embargo, esto no significa, como queda dicho, que mejoren sus oportunidades ulteriores de colocación, al menos decisivamente.

Hay que señalar aquí otro problema que afecta a la República Federal: en el marco de una de las medidas encaminadas a ofrecer una formación bien reconocida, sigue dándose el caso de que su entrenamiento se orienta a profesiones cuya perspectiva de empleo es bastante cuestionable. Puede afirmarse

${ }^{21}$ Cfr. BAETHGE y otros, «Ausbildungs- und Berufsstartprobleme von Jugendlichen unter Bedingungen verschaerfter Situationen...», Gotinga, 1980. 
desde ahora que una buena parte de los egresados de estos programas no podrán colocarse, debiendo acogerse a otros programas de reciclaje o de extensión de la formación.

\section{Puntos comunes}

Aquí como allá, los programas tratan de tener en cuenta aspectos del paro juvenil desagregados por grupos específicos. Sin embargo, en ambos países se reproducen los mismos mecanismos del mercado de trabajo; así, los débiles se instalan preponderantemente en el cuadro de medidas de más cuestionable eficacia. Se refuerza y alimenta la desventaja arrastrada en el sistema de formación, dadas las actuales condiciones del mercado de trabajo en ambos países.

En ambos países los jóvenes son sustraídos de una u otra forma del mercado de trabajo a título provisional. Las medidas pretenden dotarles de mejores oportunidades de colocación.

En ambos países la participación en los programas de los organismos laborales exigen un previo registro. Con ello quedan excluidos aquellos jóvenes que, por la razón que fuere, no se han apuntado en las oficinas correspondientes. Se da, pues, el peligro de que se refuerce la marginalidad ya congénita en determinados agregados de personas. En consecuencia, se deberían acometer estudios destinados a delimitar mejor la magnitud de tales colectivos de jóvenes. Al menos, en la República Federal se ha ilustrado convenientemente la posibilidad de una formación profesional cualificada destinada a grupos marginales del mercado de trabajo o, en general, a marginados de la sociedad global ${ }^{22}$.

La mera participación en los distintos programas de medidas no puede garantizar en ambos países un empleo ulterior. Esto cabe decir, al menos - tal como viene siendo confirmado, igualmente, por diferentes estudios-, de la mayor parte de las medidas. Por lo mismo, muchos jóvenes ven en estas ofertas bastantes razones que alimentan dudas y sospechas de arbitrariedad.

Puesto que en la República Federal de Alemania sucede a menudo que una medida sólo puede iniciarse con un grupo completo (Klassenverband, entre 15 y 20 jóvenes), los organismos laborales suelen distribuir a aquéllos más en función del número de plazas que de las aptitudes y preferencias de la persona. Todo ello alimenta el arbitrismo y la casualidad. Naturalmente, esto supone un lastre para las posibilidades de colocación ulterior.

En ambos países, las medidas diseñadas a nivel estatal se complementan con programas de las Comunidades Autónomas y de los Estados regionales. Esto indica el peso de las peculiaridades regionales, al mismo tiempo que la imposibilidad de solucionar el problema desde instancias centralistas.

${ }_{22}$ Cfr. Arbeiterwohlfahrt Bundesverband e. V, Alternative Projekte der Jugendhilfe in Berlin, Bonn, 1983. 


\section{Parecidos}

Si bien en la RFA se habla de una ofensiva de cualificación ${ }^{23}$ en beneficio de los parados, se aprecia desde ahora que la situación española y la alemana se irán pareciendo cada vez más. Tampoco en la República Federal de Alemania habrá empleo para todos los jóvenes cualificados. Ello se debe, en parte, a la preparación de éstos para actividades de dudoso futuro. Pese a haber concluido la formación, apenas se disponen de puestos de trabajo en consonancia con aquélla. Se habla entonces de una «carencia estructural de formación». Además, en momentos de crisis en el mercado de trabajo funcionan peor los mecanismos de adaptación del sistema dual de la formación profesional. Pese a que los defensores de este sistema han ensalzado sus ventajas de flexibilidad y pragmatismo, ha sido necesario recurrir a ayudas estatales para superar crisis de formación y de empleo. Tales ayudas apoyan a las empresas cuyas funciones de formación se ven complementadas por otros puestos de entrenamiento en escuelas y otros centros. El sistema dual no puede, en efecto, brindar plazas de formación suficientes.

Pese a estos parecidos, los jóvenes parados sin formación profesional concluida constituyen mayoría en la República Federal de Alemania. Este grupo es el más vulnerable en el mercado de trabajo, como queda ilustrado por la siguiente tabla ${ }^{24}$ :

Cualificación

Parados menores de 25 años sin una formación concluida. Idem con una formación concluida

Total
Absoluto

Porcentajes

\begin{tabular}{ccc}
\hline & & \\
282.440 & & 56,1 \\
220.777 & & 43,9 \\
\hline 503.217 & & 100,0 \\
\hline
\end{tabular}

Los trabajos empíricos realizados en ambos países confirman ampliamente los resultados del estudio teórico. En España pudo extraerse, además, diversas conclusiones sobre el paradero de los distintos afectados.

Los considerables riesgos que acompañan el acceso de los jóvenes de ambas nacionalidades al sistema de formación se agudizan en determinados agregados. Es preciso incluir entre éstos y de forma destacada a los escasamente cualificados y a las mujeres jóvenes. Sorprende más todavía el hecho de que,

${ }^{23}$ Sólo la Oficina Federal de Trabajo pone a disposición, con este fin y para 1987, recursos cifrados en 5.500 millones de marcos (cfr. Handelsblatt de 20-7-1987). Se calcula que los fondos adicionales de empresas y otras fuentes privadas doblan aquella cantidad en un año.

${ }^{24}$ H. Budde/K. Kцемм, Jugend, Ausbildung, Arbeit, Dusseldorf, 1987, p. 75. 
tanto desde el punto de vista cuantitativo como cualitativo del cuadro de medidas aplicado, las mujeres jóvenes se encuentran infrarrepresentadas.

Ciertamente, en Alemania el peso de hombres y mujeres en el total de participantes en los programas es casi igual. Sin embargo, se dan importantes diferencias respecto del tipo de medidas; así, las mujeres están sobrerrepresentadas en aquellos programas con peores perspectivas de empleo.

En cuanto a España, llama especialmente la atención la escasa representación de la mujer en tales programas, pese a que su participación en el conjunto de parados es tan fuerte como la de los hombres. He aquí la representación por sexo en los dos programas más importantes ofrecidos en ese país:

\section{Participantes en ambos programas por sexo (porcentajes)}

\begin{tabular}{|c|c|c|}
\hline & Hombres & Mujeres \\
\hline Contratación para la formación $\ldots \ldots \ldots$ & 66 & 34 \\
\hline Contratos en prácticas $\ldots \ldots \ldots \ldots \ldots \ldots$ & 55,5 & 44,5 \\
\hline
\end{tabular}

No pudo someterse a prueba la sospecha de que se diera aquí un mayor trasvase de la mujer a la situación de reserva potencial. Tal hipótesis podría verse respaldada por las bajas tasas de actividad femenina en los grupos de edad inferiores a los 25 años y, al mismo tiempo, por la mayor permanencia de las mujeres en los centros de formación. En esta línea deberían moverse estudios posteriores.

Dado que en el caso español no ha podido demostrarse una relación significativa entre las variables «sexo» y la prolongación de las medidas, cabe admitir, en mi opinión, que la discriminación de las chicas ha tenido lugar antes de iniciarse las medidas, es decir, en el momento de la selección de los participantes en los distintos programas. Una vez que las mujeres están incluidas en la oferta, parece que cumplen su cometido tan bien como sus colegas masculinos.

En ambos países predominan aquellas medidas que son más económicas. En cada uno de ellos se ha potenciado especialmente una medida. En España, la contratación para la formación. En la República Federal de Alemania, otra situada, asimismo, en el marco escolar; la más económica, pero de dudosa eficacia en cuanto a las posibilidades de empleo futuro.

Respecto de la calidad de las medidas estudiadas, según el grado de integración estable en el sistema de empleo, cabe hacer algunas importantes consideraciones. Hay que preguntarse si vale la pena el dispendio de recursos per- 
sonales y financieros. En lo que a España respecta, y después de concluida la aplicación de las medidas «contratación para la formación» y «contratos en prácticas», sólo un 7,5 por 100 de los jóvenes encuestados afirmaron poseer un empleo permanente y estable. Casi la mitad se encontraba de nuevo parado. En cuanto a los demás, o bien se había prolongado el programa, o bien se había encontrado una colocación temporal relacionada con otro programa.

En lo que respecta a la República Federal de Alemania -país en el que no había terminado la aplicación de las medidas en el momento de realizarse el estudio-, cabe presumir una tasa de éxito mayor (aprox., 50 por 100). En todo caso, esto pudo comprobarse mediante análisis de datos secundarios en la parte teórica del libro y teniendo en cuenta la media de todas las medidas y el propósito confesado de utilizar el programa para encontrar empleo. Sin embargo, también en la República Federal de Alemania se daban ofertas de cualificación de dudosa efectividad: por ejemplo, se forma a los jóvenes parados en oficios-profesiones que ahora mismo registran altas tasas de desempleo (así, vendedores, peluqueras, mecánicos de vehículos). En estos casos se procede a una cualificación bastante alejada de los verdaderos requisitos del mercado de trabajo.

Poniéndonos a considerar la aplicación de otras medidas, uno no puede ahorrarse la impresión de que es más importante «recoger gente de la calle» o «aliviar las estadísticas» de los organismos laborales que una adecuada y prometedora cualificación de los jóvenes.

Puesto que las medidas españolas se instalan de forma abrumadora en el sistema de empleo, existe el peligro de que los jóvenes asuman una función de relleno en ofertas tales como la de contratación para la formación. Pueden, en efecto, ser contratados temporalmente como mano de obra barata y, cuando la coyuntura afloja, ser de nuevo despedidos por las empresas sin contemplación alguna desde el punto de vista jurídico-laboral. De este modo, la cifra de ocupados puede adaptarse mejor a los vaivenes de temporada o de coyuntura. Con ello, el paro juvenil se encasquilla a un nivel prácticamente invariable pese a todas las medidas contempladas. Lo mismo cabe decir respecto de los programas alemanes orientados directamente al sector de empleo.

\section{OBSERVACIONES FINALES}

Cabe sacar las siguientes conclusiones, después de los resultados obtenidos tras las cuestiones anteriormente planteadas. En primer lugar, las medidas tomadas en ambos países sólo limitadamente pueden combatir el paro juvenil. Junto a este aspecto, los programas de ambos países tienen un significado de orden público: contribuyen provisionalmente a un alivio cuantitativo del problema. Al menos en la República Federal, se persigue aplacar un potencial 
conflictivo de política interior. Los disturbios desatados a comienzos de mayo de 1987 por jóvenes parados en Berlín muestran hasta dónde puede llegar la acumulación de tales situaciones. Berlín-Kreuzberg fue uno de esos escenarios con una fuerte participación de jóvenes parados.

No bastan, pues, ni con mucho, las ofertas hechas, que son, en alguna medida, cuestionables. Debería guardarse, precisamente en la República Federal, de una sobrevaloración de su eficacia, tanto en el tipo de cualificación como en la política de formación. También en España, el paro juvenil es indudablemente parte de una profunda crisis generalizada del sistema de empleo, que no puede solucionarse sólo a base de estrategias de cualificación forzadas.

Cabe admitir, pues, que los esfuerzos españoles son más honrados, al situarse preponderantemente en el sistema de empleo. Ciertamente, en España se necesita una formación profesional, dado que los cambios estructurales en el mundo del trabajo (nuevas tecnologías) exigen nuevas cualificaciones de la fuerza de trabajo ${ }^{25}$. Con todo, ello no constituye una salida a la crisis de empleo. En ambos países siempre ha habido y habrá dificultades de acoplamiento entre los sistemas de empleo y de educación. Esta discrepancia estructural tiene un componente básico neutral a la coyuntura, es decir, las aludidas dificultades de acoplamiento pueden surgir en diferentes estadios de la actividad económica de un país. Por ello, tampoco pueden aclararse causalmente las concatenaciones que provocan crisis de empleo.

En consecuencia, el empleo debe tener prioridad frente a la formación en el diseño de medidas eficaces. Por lo mismo, es preciso hacer algún giro im. portante, especialmente en Alemania. Naturalmente, de poco servirá un cuadro de medidas en espacios carentes hasta el momento de condiciones-marco legales, organizativas y administrativas para fomentar el empleo. En ambos países el sector público debería redoblar sus esfuerzos para diseñar el futuro.

Puesto que el problema deI paro juvenil es, ante todo, parte de una crisis envolvente, la del empleo, las medidas que apliquen ambos países en la solución del paro no pueden instalarse únicamente entre determinados grupos dentro del mercado de trabajo. Se invoca la actuación del Estado y de empresarios y sindicatos.

Se ha podido comprobar ya en la República Federal de Alemania que una reducción de la(s) jornada(s) laboral(es) ha supuesto un aumento de puestos de trabajo. Aunque no hay que ver en ello la panacea - puesto que el tiempo de trabajo reducido se compensa parcialmente por nuevos aumentos de las tasas de productividad-, tales políticas van en la buena dirección.

Por lo demás, en lo sucesivo debe plantearse con toda claridad la cues-

3. El Instituto Federal de Formación Profesional (BIBB) diseña actualmente un modelo relacionado con la cualificación profesional de jóvenes desfavorecidos y teniendo en cuenta el impacto de las nuevas tecnologías. El autor es asesor científico de dicho proyecto. 
tión de si una financiación pública de puestos de trabajo conviene más que una financiación del paro o de diversas medidas estatales de dudosa eficacia. Unos primeros cálculos efectuados en Alemania indican que, para determinados grupos, la financiación de la ocupación es a la larga menos costosa que la del paro ${ }^{26}$. En todo caso, el sector público tiene ante sí una gran tarea en obras públicas (por ejemplo, protección de monumentos), ecología y servicios sociales.

En ambos países pueden registrarse proyectos pioneros (por ejemplo, iniciativas de empleo comunitarias, programas del mismo tipo) cuya efectividad y éxito deberá examinarse más tarde con el fin de trasladar a otros medios la experiencia acumulada.

(Traducción de María Guiomar Garmendia Pérez.)

${ }^{26}$ BBJ-Consult, Programm zur Beschaeftigung und Qualifizierung von Langzeitarbeitslosen und Sozialbilfeempfaengern, Berlín, 1986. 